\title{
An Analysis on Single and Central Diffractive Heavy Flavour Production at Hadron Colliders
}

\author{
M. V. T. Machado \\ Universidade Federal do Pampa, Centro de Ciências Exatas e Tecnológicas \\ Campus de Bagé, Rua Carlos Barbosa, CEP 96400-970, Bagé, RS, Brazil
}

(Received on 20 March, 2008)

\begin{abstract}
In this contribution results from a phenomenological analysis for the diffractive hadroproduction of heavy flavors at high energies are reported. Diffractive production of charm, bottom and top are calculated using the Regge factorization, taking into account recent experimental determination of the diffractive parton density functions in Pomeron by the H1 Collaboration at DESY-HERA. In addition, multiple-Pomeron corrections are considered through the rapidity gap survival probability factor. We give numerical predictions for single diffractive as well as double Pomeron exchange (DPE) cross sections, which agree with the available data for diffractive production of charm and beauty. We make estimates which could be compared to future measurements at the LHC.

Keywords: Heavy flavour production; Pomeron physics; Single diffraction; Quantum Chromodynamics
\end{abstract}

\section{INTRODUCTION}

For a long time, diffractive processes in hadron collisions have been described by Regge theory in terms of the exchange of a Pomeron with vacuum quantum numbers [1]. However, the nature of the Pomeron and its reaction mechanisms are not completely known. Currently, a promising way to clarify these questions is using the hard scattering to resolve the quark and gluon content in the Pomeron [2], regarding that a parton structure is natural in a modern QCD approach to the strongly interacting Pomeron. Form the experimental point of view, observations of diffractive deep inelastic scattering (DDIS) at HERA have increased the knowledge about the QCD Pomeron, where its diffractive distributions of singlet quarks and gluons have been phenomenologically determined [3].

In hadronic collisions, a single diffractive event is characterized by one of the colliding hadrons emitting a Pomeron that scatters off the other hadron. Hard diffractive events with a large momentum transfer are also set by the absence of hadronic energy in certain angular regions of the final state phase space (large rapidity gaps). For events in which both colliding hadrons remain intact as they each emit a Pomeron we have the so-called central diffractive events. In the latter case, also known as double Pomeron exchange (DPE) processes, both incoming hadrons are quasi-elastically scattered and the final states system in the center region is produced by Pomeron-Pomeron interaction.

Here, we concentrate on the single diffractive processes $p+p(\bar{p}) \rightarrow p+J / \Psi[\Upsilon]+X$ and the central diffractive reactions, $p+p(\bar{p}) \rightarrow p+J / \Psi[\Upsilon]+p(\bar{p})$. The diffractive heavy quarkonium production has drawn attention because their large masses provide a natural scale to guarantee the application of perturbative QCD. There are several mechanisms proposed for the quarkonium production in hadron colliders $[4,5]$, as the color singlet model, the color octet model and the color evaporation model. An important feature of these perturbative QCD models is that the cross section for quarkonium production is expressed in terms of the product of two gluon densities at large energies. This feature is transferred to the diffractive quarkonium production, which is now sensitive to the gluon content of the Pomeron at small- $x$ and may be particularly useful in studying the different mechanisms for quarkonium production.

In order to do so, we will use the hard diffractive factorization, where the diffractive cross section is the convolution of diffractive parton distribution functions and the corresponding diffractive coefficient functions. At high energies there are important contributions from unitarization effects to the singlePomeron exchange cross section. These absorptive corrections cause the suppression of any large rapidity gap process, except elastic scattering. In the black disk limit the absorptive corrections may completely terminate those processes. This partially occurs in (anti)proton-proton collisions, where unitarity is nearly saturated at small impact parameters [6]. These multiple-Pomeron contributions depends on the particular hard process and it is called survival probability factor, which are important for the reliability of theory predictions.

The present contribution is organized as follows. In next section, we present the main formulas to compute the inclusive and diffractive cross sections for heavy quarkonium hadroproduction. We also present the parameterization for the diffractive partons distribution in the Pomeron, extracted recently in DESY-HERA, and theoretical estimations for the gap survival probability factor. In the last section we present the numerical results for Tevatron and perform predictions to future measurements at the LHC experiment. The compatibility with data is analyzed and the comparison with other approaches is considered. As an anticipation of the main results, at the Tevatron the single and central diffractive $J / \Psi$ and $\Upsilon$ production are observable with a single diffractive ratio $R^{\mathrm{SD}}$ (Tevatron) that is between $1 \%$ (charmonium) and 0.5 $\%$ (bottomonium), with lower values at the LHC. Also, it is predicted that $R_{\text {theory }}^{J / \Psi}=1.12 \pm 0.19 \%$. The central diffractive cross sections are still feasible to be measured, despite the very small diffractive ratios. Therefore, we stress that the theoretical model dependence is either large and more detailed studies are timely. 


\section{DIFFRACTIVE HADROPRODUCTION OF HEAVY QUARKONIUM}

For our purpose we will use the Color Evaporation Model (CEM) [7]. The main reasons for this choice are its simplicity and fast phenomenological implementation, which are the base for its relative success in describing high energy data[4, 5]. In such an approach, the cross section for a process in which partons of two hadrons, $h_{1}$ and $h_{2}$, interact to produce a heavy quarkonium state, $h_{1}+h_{2} \rightarrow H\left(n J^{\mathrm{CP}}\right)+X$, is given by the cross section of open heavy-quark pair production that is summed over all spin and color states. All information on the non-perturbative transition of the $Q \bar{Q}$ pair to the heavy quarkonium $H$ of quantum numbers $J^{\mathrm{PC}}$ is contained in the factor $F_{n J} J^{\mathrm{PC}}$ that a priori depends on all quantum numbers [7],

$$
\sigma\left(h_{1} h_{2} \rightarrow H\left[n J^{\mathrm{CP}}\right] X\right)=F_{n J^{\mathrm{PC}}} \bar{\sigma}\left(h_{1} h_{2} \rightarrow Q \bar{Q} X\right),
$$

where $\bar{\sigma}(Q \bar{Q})$ is the total hidden cross section of open heavyquark production calculated by integrating over the $Q \bar{Q}$ pair mass from $2 m_{Q}$ to $2 m_{O}$, with $m_{O}$ is the mass of the associated open meson. The hidden cross section can be obtained from the usual expression for the total cross section to NLO. These hadronic cross sections in $p p$ collisions can be written as

$$
\begin{aligned}
\sigma_{p p}\left(\sqrt{s}, m_{Q}^{2}\right) & =\sum_{i, j=q, \bar{q}, g} \int d x_{1} d x_{2} f_{i}^{p}\left(x_{1}, \mu_{F}^{2}\right) f_{j}^{p}\left(x_{2}, \mu_{F}^{2}\right) \\
& \times \widehat{\sigma}_{i j}\left(\sqrt{s}, m_{Q}^{2}, \mu_{F}^{2}, \mu_{R}^{2}\right)
\end{aligned}
$$

where $x_{1}$ and $x_{2}$ are the fractional momenta carried by the colliding partons and $f_{i}^{p}$ are the proton parton densities. The partonic cross sections are known up to NLO accuracy [8]. Here, we assume that the factorization scale, $\mu_{F}$, and the renormalization scale, $\mu_{R}$, are equal. We also take $\mu=2 m_{Q}$, using the quark masses $m_{c}=1.2 \mathrm{GeV}$ and $m_{b}=4.75 \mathrm{GeV}$, which provide an adequate description of open heavy-flavour production [8]. The invariant mass is integrated over $4 m_{c}^{2} \leq \hat{s} \leq 4 m_{D}^{2}$ in the charmonium case and $4 m_{b}^{2} \leq \hat{s} \leq 4 m_{B}^{2}$ for $\Upsilon$ production. The factors $F_{n J} \mathrm{PC}$ are experimentally determined [9] to be $F_{11^{--}} \approx 2.5 \times 10^{-2}$ for $J / \Psi$ and $F_{11^{--}} \approx 4.6 \times 10^{-2}$ for $\Upsilon$. These coefficients are obtained with NLO cross sections for heavy quark production [9].

The agreement with the total cross section data is fairly good. The low $x$ region is particularly relevant for $J / \Psi$ production at the LHC as well as at Tevatron. For charmonium production, the $g g$ process becomes dominant and information on the gluon distribution is of particular importance. In Fig. 1, the differential cross section $\left.\frac{d \sigma_{p N \rightarrow J / \Psi}}{d y}\right|_{y=0}$ is shown as a function of center of mass energy, $\sqrt{s}$. The Tevatron data indicates that correction for gluon saturation may be important in bringing the theoretical curve closer to the experimental result. In particular, the typical values of Bjorken-x would be $x \sim 2 m_{c} / \sqrt{s} \approx 7 \times 10^{-4}$ for Tevatron and $x \approx 10^{-4}$ at the LHC taken at a relatively low momentum scale $Q^{2}=m_{\Psi}^{2}=9$ $\mathrm{GeV}^{2}$. In Fig. 2, the differential cross section $B \times\left.\frac{d \sigma_{p N \rightarrow \mathrm{\Upsilon}}}{d y}\right|_{y=0}$ is presented as a function of the center of mass energy (solid line). In addition, the measured cross sections for the sum of the three $\Upsilon$ states $\left(\Upsilon+\Upsilon^{\prime}+\Upsilon^{\prime \prime}\right)$ in the dilepton decay channel are shown [4]. The agreement of the CEM model with accelerator data is very good and an extrapolation to the LHC is presented. The results give us considerable confidence in the extrapolation to the LHC energy.

\subsection{Diffractive cross section - single-Pomeron exchange}

For the hard diffractive processes we will consider the Ingelman-Schlein (IS) picture [2], where the Pomeron structure (quark and gluon content) is probed. In the case of single diffraction, a Pomeron is emitted by one of the colliding hadrons. That hadron is detected, at least in principle, in the final state and the remaining hadron scatters off the emitted Pomeron. The diffractive cross section of a hadron-hadron collision is assumed to factorise into the total Pomeronhadron cross section and the Pomeron flux factor [2]. The single diffractive event, $h_{1}+h_{2} \rightarrow h_{1,2}+H\left[n J^{\mathrm{CP}}\right]+X$, may then be written as

$$
\begin{aligned}
& \frac{d \sigma^{\mathrm{SD}}\left(h_{i}+h_{j} \rightarrow h_{i}+H\left[n J^{\mathrm{CP}}\right]+X\right)}{d x_{\mathrm{IP}}^{(i)} d\left|t_{i}\right|}= \\
& F_{n J} \mathrm{PC} \times f_{\mathrm{IP} / h_{i}}\left(x_{\mathrm{IP}}^{(i)},\left|t_{i}\right|\right) \bar{\sigma}\left(\mathrm{IP}+h_{j} \rightarrow Q \bar{Q}+X\right),
\end{aligned}
$$

where the Pomeron kinematical variable $x_{\mathrm{IP}}$ is defined as $x_{\mathrm{IP}}^{(i)}=s_{\mathrm{IP}}^{(j)} / s_{i j}$, where $\sqrt{s_{\mathrm{IP}}^{(j)}}$ is the center-of-mass energy in the Pomeron-hadron $j$ system and $\sqrt{s_{i j}}=\sqrt{s}$ the centerof-mass energy in the hadron $i$-hadron $j$ system. The momentum transfer in the hadron $i$ vertex is denoted by $t_{i}$. A similar factorization can also be applied to central diffraction, where both colliding hadrons can in principle be detected in the final state. The central quarkonium production, $h_{1}+h_{2} \rightarrow h_{1}+H\left[n J^{\mathrm{CP}}\right]+h_{2}$, is characterized by two quasielastic hadrons with rapidity gaps between them and the central heavy quarkonium products. The central diffractive cross section may then be written as,

$$
\begin{aligned}
& \frac{d \sigma^{\mathrm{CD}}\left(h_{i}+h_{j} \rightarrow h_{i}+H\left[n J^{\mathrm{CP}}\right]+h_{j}\right)}{d x_{\mathrm{IP}}^{(i)} d x_{\mathrm{IP}}^{(j)} d\left|t_{i}\right| d\left|t_{j}\right|}=F_{n J^{\mathrm{PC}}} \times \\
& f_{\mathrm{IP} / i}\left(x_{\mathrm{IP}}^{(i)},\left|t_{i}\right|\right) f_{\mathrm{IP} / j}\left(x_{\mathrm{IP}}^{(j)},\left|t_{j}\right|\right) \bar{\sigma}(\mathrm{IP}+\mathrm{IP} \rightarrow Q \bar{Q}+X) .
\end{aligned}
$$

Here, we assume that one of the hadrons, say hadron $h_{1}$, emits a Pomeron whose partons interact with partons of the hadron $h_{2}$. Thus the parton distribution $x_{1} f_{i / h_{1}}\left(x_{1}, \mu^{2}\right)$ is replaced by the convolution between a distribution of partons in the Pomeron, $\beta f_{a / \mathrm{IP}}\left(\beta, \mu^{2}\right)$, and the "emission rate" of Pomerons by the hadron, $f_{\mathrm{IP} / h}\left(x_{\mathrm{IP}}, t\right)$. The last quantity, $f_{\mathrm{IP} / h}\left(x_{\mathrm{IP}}, t\right)$, is the Pomeron flux factor and its explicit formulation is described in terms of Regge theory. Therefore, we can rewrite the parton distribution as

$$
\begin{aligned}
x_{1} f_{a / h_{1}}\left(x_{1}, \mu^{2}\right) & =\int d x_{\mathrm{IP}} \int d \beta \int d t f_{\mathrm{IP} / h_{1}}\left(x_{\mathrm{IP}}, t\right) \\
& \times \beta f_{a / \mathrm{IP}}\left(\beta, \mu^{2}\right) \delta\left(\beta-\frac{x_{1}}{x_{\mathrm{IP}}}\right),
\end{aligned}
$$




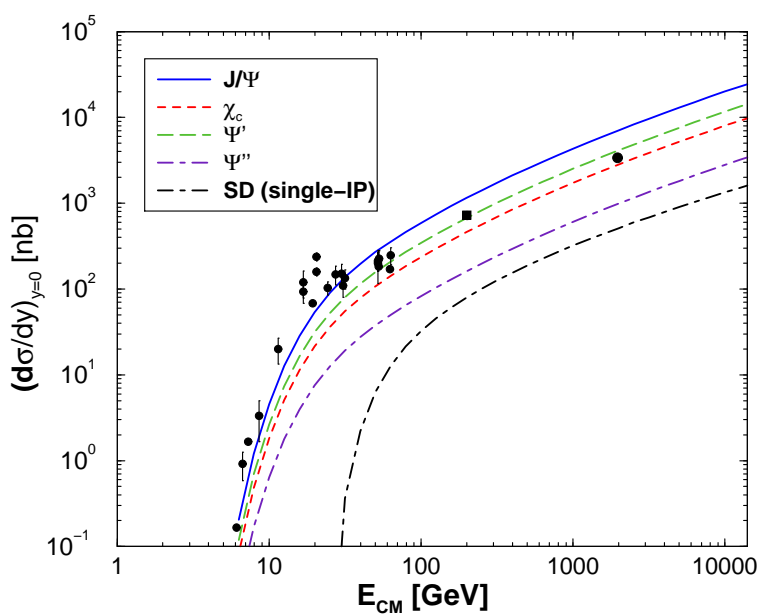

FIG. 1: The differential cross section $d \sigma /\left.d y\right|_{y=0}$ as a function of energy for the $J / \Psi$ production (solid line). Single diffractive cross section (dot-dashed line) and accelerator data are also shown (see text).

Using the substitution given in Eq. (4), the hidden heavy flavour cross section can be obtained from Pomeron-hadron cross sections for single and central diffraction processes,

$$
\begin{gathered}
\frac{d \sigma(\mathbb{P}+h \rightarrow Q \bar{Q}+X)}{d x_{1} d x_{2}}=\sum_{i, j=q \bar{q}, g} \frac{f_{i / \mathbb{I P}}\left(x_{1} / x_{\mathrm{IP}}^{(1)} ; \mu_{F}^{2}\right)}{x_{\mathrm{IP}}^{(1)}} \\
\times f_{j / h_{2}}\left(x_{2}, \mu_{F}^{2}\right) \hat{\sigma}_{i j}\left(\hat{s}, m_{Q}^{2}, \mu_{F}^{2}, \mu_{R}^{2}\right)+(1 \rightleftharpoons 2),
\end{gathered}
$$

and

$$
\begin{aligned}
& \frac{d \sigma(\mathbb{P}+\mathbb{P} \rightarrow Q \bar{Q}+X)}{d x_{1} d x_{2}}=\sum_{i, j=q \bar{q}, g} \frac{f_{i / \mathbb{I P}}\left(x_{1} / x_{\mathrm{IP}}^{(1)} ; \mu_{F}^{2}\right)}{x_{\mathrm{IP}}^{(1)}} \\
& \times \frac{f_{j / \mathbb{P}}\left(x_{2} / x_{\mathrm{IP}}^{(2)} ; \mu_{F}^{2}\right)}{x_{\mathrm{IP}}^{(2)}} \hat{\sigma}_{i j}\left(\hat{s}, m_{Q}^{2}, \mu_{F}^{2}, \mu_{R}^{2}\right) .
\end{aligned}
$$

In the numerical calculations, we will consider the diffractive pdf's recently obtained by the $\mathrm{H} 1$ Collaboration at DESYHERA [3]. The Pomeron structure function has been modeled in terms of a light flavour singlet distribution $\Sigma(z)$, consisting of $u, d$ and $s$ quarks and anti-quarks and a gluon distribution $g(z)$. The Pomeron carries vacuum quantum numbers, thus it is assumed that the Pomeron quark and antiquark distributions are equal and flavour independent: $q_{\mathrm{IP}}^{f}=\bar{q}_{\mathrm{IP}}^{f}=\frac{1}{2 N_{f}} \Sigma_{\mathrm{IP}}$, where $\Sigma_{\mathrm{IP}}$ is a Pomeron singlet quark distribution and $N_{f}$ is the number of active flavours. Moreover, for the Pomeron flux factor, introduced in Eq. (4), we take the experimental analysis of the diffractive structure function [3], where the $x_{I P}$ dependence is parameterized using a flux factor motivated by Regge theory [1],

$$
f_{\mathrm{IP} / \mathrm{p}}\left(x_{\mathrm{IP}}, t\right)=A_{\mathrm{IP}} \cdot \frac{e^{B_{\mathrm{IP}} t}}{x_{\mathrm{IP}}^{2 \alpha_{\mathrm{IP}}(t)-1}},
$$

where the Pomeron trajectory is assumed to be linear, $\alpha_{\mathrm{IP}}(t)=$ $\alpha_{I P}(0)+\alpha_{I P}^{\prime} t$.

\subsection{Multiple-Pomeron exchange corrections}

Here, we consider the suppression of the hard diffractive cross section by multiple-Pomeron scattering effects. This is taken into account through a gap survival probability factor, $<|S|^{2}>$, which can be described in terms of screening or absorptive corrections [10]. This suppression factor of a hard process accompanied by a rapidity gap depends not only on the probability of the initial state survive, but is sensitive to the spatial distribution of partons inside the incoming hadrons, and thus on the dynamics of the whole diffractive part of the scattering matrix. The survival factor of a large rapidity gap (LRG) in a hadronic final state is the probability of a given LRG not be filled by debris, which originate from the soft rescattering of the spectator partons and/or from the gluon radiation emitted by partons taking part in the hard interaction. Let $\mathcal{A}(s, b)$ be the amplitude of the particular diffractive process of interest, considered in the impact parameter, $b$, space. Therefore, the probability that there is no extra inelastic interaction is

$$
<|S|^{2}>=\frac{\int d^{2} b|\mathcal{A}(s, b)|^{2} \exp [-\Omega(s, b)]}{\int d^{2} b|\mathcal{A}(s, b)|^{2}},
$$

where $\Omega$ is the opacity (or optical density) of the interaction.

We will consider the theoretical estimates for $\left\langle|S|^{2}>\right.$ from Ref. [11] (labeled KMR), which considers a two-channel eikonal model and rescattering effects. The survival probability factor is computed for single, central and double diffractive processes at several energies, assuming that the spatial distribution in impact parameter space is driven by the slope $B$ of the pomeron-proton vertex. We will consider the results for single diffractive processes with $2 B=5.5 \mathrm{GeV}^{-2}$ (slope of the electromagnetic proton form factor) and without $N^{*}$ excitation, which is relevant to a forward proton spectrometer (FPS) measurement. Thus, we have $\left\langle|S|^{2}>_{\mathrm{KMR}}^{\mathrm{SD}}=0.15,[0.09]\right.$ and $<|S|^{2}>{ }_{\mathrm{KMR}}^{\mathrm{CD}}=0.08,[0.04]$ for $\sqrt{s}=1.8 \mathrm{TeV}$ (Tevatron) $[\sqrt{s}=$ $14 \mathrm{TeV}$ (LHC)]. There are similar theoretical estimates, as the GLM approach [12], which also considers a multi-channel eikonal approach.

\section{RESULTS AND SUMMARY}

For the numerical calculations, the new $\mathrm{H} 1$ parameterization for the diffractive pdf's [3] has been used. The 'H1 2006 DPDF Fit A' is considered, with the cut $x_{\mathbb{P}}<0.1$. The singlePomeron results are presented in Figs. 1 and 2 for $J / \Psi$ and $\Upsilon$ production (dot-dashed curves), respectively. The single diffractive contribution is large, being of order 5-6\% from the inclusive cross section. The results corrected by multiplePomeron suppression factor are a factor about 1/10 lower than the single-Pomeron ones. It should be stressed that sizable uncertainties are introduced by changing, for instance, quark 


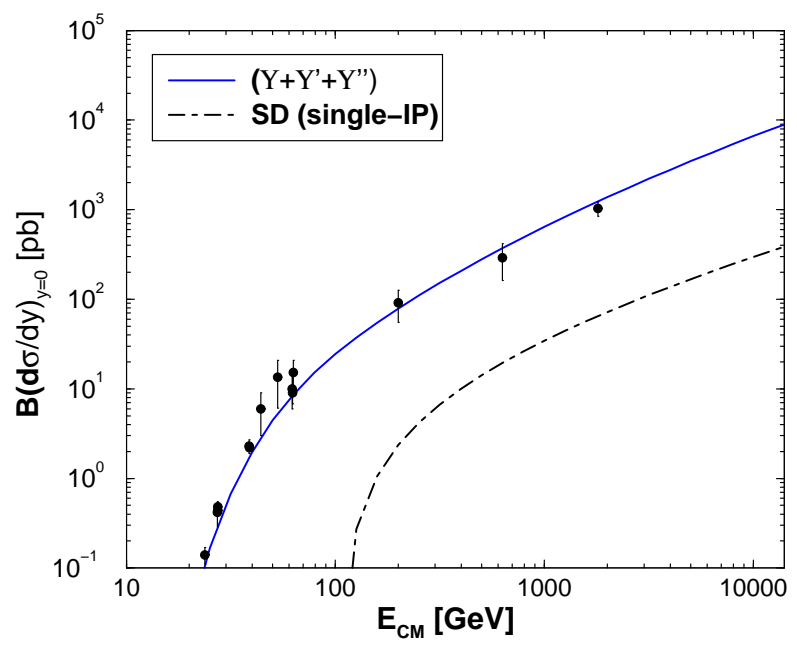

FIG. 2: The differential cross section $B \times d \sigma /\left.d y\right|_{y=0}$ as a function of energy for the $\Upsilon+\Upsilon^{\prime}+\Upsilon^{\prime \prime}$ production (solid line). Single diffractive cross section (dot-dashed line) and accelerator data are also shown (see text)

masses and/or the renormalization scale. However, our purpose here is to estimate the diffractive ratios $\sigma^{D} / \sigma_{\text {tot }}$, which are less sensitive to a particular choice. For sake of illustration, we have $\left.\frac{d \sigma(J / \Psi)}{d y}\right|_{y=0} ^{\mathrm{SD}}=85(566) \mathrm{nb}$ for the energy of $\sqrt{s}=2 \mathrm{TeV}$ and $159(1770) \mathrm{nb}$ for LHC energy, $\sqrt{s}=14 \mathrm{TeV}$. For $\Upsilon$ we have $\left.B \frac{d \sigma(\Upsilon)}{d y}\right|_{y=0} ^{\mathrm{SD}}=11(72) \mathrm{pb}$ and $35(386) \mathrm{pb}$ for Tevatron and LHC, respectively. Numbers between parentheses correspond to values not corrected by survival probability gap factor.

Concerning the central diffractive cross-sections, the predictions give small values. For instance, we have $\left.\frac{d \sigma(J / \Psi)}{d y}\right|_{y=0} ^{\mathrm{CD}}=18 \mathrm{nb}$ and $\left.B \frac{d \sigma(\Upsilon)}{d y}\right|_{y=0} ^{\mathrm{CD}}=0.8 \mathrm{pb}$ at $\sqrt{s}=2 \mathrm{TeV}$. This gives little room to observe central diffractive $\Upsilon$ events at the Tevatron but could be promising for the LHC. The values reach $\left.\frac{d \sigma(J / \Psi)}{d y}\right|_{y=0} ^{\mathrm{CD}}=45 \mathrm{nb}$ and $\left.B \frac{d \sigma(\Upsilon)}{d y}\right|_{y=0} ^{\mathrm{CD}}=3 \mathrm{pb}$ at $\sqrt{s}=14$ $\mathrm{TeV}$. Once again, the results corrected by multiple-Pomeron suppression factor are a factor about $1 / 10$ lower than the usual IS model.

Let us now compute the diffractive ratios. We have defined the single diffractive ratio as $R_{\mathrm{SD}}=\frac{d \sigma}{d y}^{\mathrm{SD}} / \frac{d \sigma}{d y}^{\text {inc }}$ and the central diffractive ratio as $R_{\mathrm{CD}}=\frac{d \sigma}{d y}^{\mathrm{CD}} / \frac{d \sigma}{d y}^{\text {inc }}$. The results are summarized in Table I, where the diffractive ratios for heavy quarkonium production are presented for Tevatron and LHC energies. The multiple-Pomeron correction factors are taken from KMR model. The numbers between parentheses represent the single-Pomeron calculation. Based on these results we verify that the $J / \Psi$ and $\Upsilon$ production in single diffractive process could be observable in Tevatron and LHC, with a diffractive ratio of order of $1 \%$ or less. This is a similar ratio measured in $W$ and $Z$ production at the Tevatron [14]. The predictions for central diffractive scattering are still not very promising, giving small ratios. However, the study of these events is worthwhile since their experimental signals
TABLE I: Model predictions for single and central diffractive quarkonium production in Tevatron and the LHC. Numbers between parentheses represent the estimates using the single Pomeron exchange.

\begin{tabular}{lcll}
\hline \hline$\sqrt{s}$ & Quarkonium & $R_{\mathrm{SD}}(\%)$ & $R_{\mathrm{CD}}(\%)$ \\
\hline $2.0 \mathrm{TeV}$ & $J / \Psi$ & $0.93(6.2)$ & $0.2(2.5)$ \\
$14 \mathrm{TeV}$ & $J / \Psi$ & $0.50(5.9)$ & $0.15(3.7)$ \\
$2.0 \mathrm{TeV}$ & $\left(\Upsilon+\Upsilon^{\prime}+\Upsilon^{\prime \prime}\right)$ & $0.78(5.2)$ & $0.06(0.7)$ \\
$14 \mathrm{TeV}$ & $\left(\Upsilon+\Upsilon^{\prime}+\Upsilon^{\prime \prime}\right)$ & $0.39(4.3)$ & $0.03(0.8)$ \\
\hline \hline
\end{tabular}

are quite clear. Finally, our theoretical prediction is in good agreement with the experimental measurement of CDF [13], which found $R_{\mathrm{SD}}^{J / \Psi}=1.45 \pm 0.25 \%$. As the main theoretical uncertainty in determining the diffractive ratio is the value for the survival factor, we consider the theoretical band 0.210.15 for Tevatron energy. Therefore, our prediction gives $R_{\text {theory }}^{J / \Psi}=1.12 \pm 0.19 \%$, which is consistent with the Tevatron determination.

Our calculation can be compared to available literature in diffractive heavy quarkonium production. For instance, in Ref. [15] the large $p_{T} J / \Psi$ production in hard diffractive process is computed using the color octet fragmentation mechanism and the normalized Pomeron flux [16]. They found a SD $J / \Psi$ cross section at large $p_{T}(\geq 8 \mathrm{GeV})$ of order $10 \mathrm{pb}$ and a diffractive ratio $R_{\mathrm{SD}}=0.65 \pm 0.15 \%$. This is closer to our calculation for the SD ratio for Tevatron within the theoretical errors. Afterwards, in Ref. [17] the color-octet mechanism combined with the two gluon exchange model(in LO approximation in QCD) for the diffractive $J / \Psi$ production is considered. Now, the SD cross section is $\sigma(p \bar{p} \rightarrow J / \Psi X)=66$ $\mathrm{nb}$. The comparison between these calculations shows the size of the large theoretical uncertainty. A related calculation, the $J / \Psi+\gamma$ diffractive production, appeared in Ref. [18] based on IS model (with normalized flux [16]) and factorization formalism of NRQCD for quarkonia production. They found $\sigma(p \bar{p} \rightarrow[J / \Psi+\gamma] X)=3.0 \mathrm{pb}(8.5 \mathrm{pb})$ and diffactive ratio $R_{\mathrm{SD}}=0.5(0.15) \%$ in central region at the Tevatron (LHC). These results are somewhat still compatible with present calculation.

Concerning central diffraction, there are some theoretical studies in literature. In Ref. [19], the DPE process, $p+p(\bar{p}) \rightarrow p+\chi_{J}+p(\bar{p})$, is calculated using two-gluon exchange model in perturbative QCD. It is found the following DPE cross sections: $\sigma\left(\chi_{c 0}\right)=735 \mathrm{nb}$ and $\sigma\left(\chi_{b 0}\right)=0.88$ nb. In the same work, it is found that $\frac{d \sigma(J / \Psi+\gamma)}{d y d p_{T}}=2 \mathrm{nb} / \mathrm{GeV}$ and $\frac{d \sigma(\Upsilon+\gamma)}{d y d p_{T}}=0.5 \mathrm{pb} / \mathrm{GeV}$. Recently, in Ref. [20] the doublediffractive production of $\chi_{c}$ and $\chi_{b}$ mesons has been studied using also the Regge formalism and pQCD (including unitarity corrections). They found $d \sigma /\left.d y\right|_{y=0}=130(340) \mathrm{nb}$ for $\chi_{c}$ production and $d \sigma /\left.d y\right|_{y=0}=0.2(0.6) \mathrm{nb}$ for $\chi_{b}$ production for Tevatron (LHC). It was verified that the exclusive production is a factor 10 larger than the inclusive rate. Finally, the diffractive $\chi$ meson production bas been computed [21] using the Bialas-Landshoff formalism and makes use of the DPEMC Monte-Carlo simulation for small-mass 
diffractive production. They found $R_{\mathrm{CD}}^{\chi_{c}}=6.5(1.6) \%$ and $R_{\mathrm{CD}}^{\chi_{b}}=22(1.83) \%$ for Tevatron (LHC). In summary, the theoretical predictions for exclusive meson production are still quite distinct and more detailed studies are deserved.

In summary, we have presented predictions for diffractive heavy quarkonium production at the Tevatron and the LHC. We use Regge factorization, corrected by unitarity corrections modeled by a gap survival probability factor (correction for multiple-Pomeron exchange). The perturbative formalism for meson hadroproduction is based on the CEM model, which is quite successful in describing experimental results for inclusive production. For the Pomeron structure function, recent $\mathrm{H} 1$ diffractive parton density functions have been used. The results are directly dependent on the quark singlet and gluon content of the Pomeron. We estimate the multiple interaction corrections taking the theoretical prediction a multichannel model (KMR), where the gap factor decreases on energy. That is, $\left\langle|S|^{2}\right\rangle \simeq 15 \%$ for Tevatron energies going down to $<|S|^{2}>\simeq 9 \%$ at LHC energy (for single diffractive process). We found that at the Tevatron single and central diffractive $J / \Psi$ and $\Upsilon$ production is observable with a single diffractive ratio $R^{\mathrm{SD}}$ (Tevatron) that is between $1 \%$ (charmonium) and $0.5 \%$ (bottomonium), with lower values at the LHC. In particular, we predict $R_{\text {theory }}^{J / \Psi}=1.12 \pm 0.19 \%$, which is in agreement with Tevatron measurements. The central diffractive cross sections for quarkonium production are still feasible to be measured, despite the very small diffractive ratios. In this case, the theoretical model dependence is still very large and detailed studies are deserved.

\section{Acknowledgments}

This work was supported by $\mathrm{CNPq}$, Brazil. The author is grateful to Uri Maor and David dEnterria for useful discussions.
[1] P. D. B. Collins, An Introduction to Regge theory and high energy physics (Cambridge University Press, Cambridge, England, 1977).

[2] G. Ingelman and P. E. Schlein, Phys. Lett. B 152, 256 (1985)

[3] H1 Collab., A. Aktas et al., Eur. Phys. J. C 48, 715 (2006).

[4] J. P. Lansberg, Int. J. Mod. Phys. A 21, 3857 (2006).

[5] M. Krämer, Prog. Part. Nucl. Phys. 47, 141 (2001).

[6] B. Z. Kopeliovich, I. K. Potashnikova, B. Povh, and E. Predazzi, Phys. Rev. Lett. 85, 507 (2000); Phys. Rev. D 63, 054001 (2001).

[7] J. F. Amundson, O. J. P. Eboli, E. M. Gregores, and F. Halzen, Phys. Lett. B 390, 323 (1997); C. Brenner Mariotto, M. B. Gay Ducati, and G. Ingelman, Eur. Phys. J. C 23, 527 (2002).

[8] M. L. Mangano, P. Nason, and G. Ridolfi, Nucl. Phys. B 373 , 295 (1992); A. Petrelli, M. Cacciari, M. Greco, F. Maltoni, and M. L. Mangano, Nucl. Phys. B 514, 245 (1998); S. Frixione et al., Adv. Ser. Direct High Energy Phys. 15, 607 (1998).

[9] G. A. Schuler and R. Vogt, Phys. Lett. B 387, 181 (1996).

[10] J. D. Bjorken, Int. J. Mod. Phys. A 7, 4189 (1992); Phys. Rev. D 47, 101 (1993).
[11] A. B. Kaidalov, V. A. Khoze, A. D. Martin, and M. G. Ryskin, Eur. Phys. J. C 21, 521 (2001).

[12] E. Gotsman, E. Levin, U. Maor, and A. Prygarin, arXiv:hep$\mathrm{ph} / 0511060$.

[13] A. A. Affolder et al. [CDF Collaboration], Phys. Rev. Lett. 87, $241802(2001)$.

[14] M. B. Gay Ducati, M. M. Machado, and M. V. T. Machado, Phys. Rev. D 75, 114013 (2007).

[15] F. Yuan and K. T. Chao, Phys. Rev. D 57, 5658 (1998).

[16] K. Goulianos, Phys. Lett. B 358, 379 (1995).

[17] F. Yuan, J. S. Xu, H. A. Peng, and K. T. Chao, Phys. Rev. D 58, 114016 (1998).

[18] J. S. Xu and H. A. Peng, Phys. Rev. D 59, 014028 (1999).

[19] F. Yuan, Phys. Lett. B 510, 155 (2001).

[20] V. A. Khoze, A. D. Martin, M. G. Ryskin, and W. J. Stirling, Eur. Phys. J. C 35, 211 (2004).

[21] M. Rangel, C. Royon, G. Alves, J. Barreto, and R. Peschanski, Nucl. Phys. B 774, 53 (2007). 